\title{
Endoradiosonde study of propulsion and pressure activity induced by test meals, Prostigmine, and diphenoxylate in the small intestine
}

\author{
FRANZ BÁRÁNY AND BERTIL JACOBSON \\ From the Medical Department II, St. Erik's Hospital, Stockholm, \\ and the Department of Medical Electronics, Karolinska Institute, Stockholm
}

EDITORIAL SYNOPSIS This is a study of the motility of the normal small intestine during the passage of a motorized radio capsule. Observations were made on the effect of test meals, Prostigmine, and diphenoxylate.

Studies of the motility of the human small intestine with different techniques have shown that several types of pressure waves are involved. While there is general agreement on the characteristics of these waves, their physiological significance is not entirely clear, as has recently been pointed out in Connell's (1961) review of the work in this field.

The object of the study reported in this article was to examine the possible correlation between intraluminal pressure activities and propulsion through the small intestine by simultaneous observation of these two parameters. To detect any such relationship, test meals and two drugs, Prostigmine and diphenoxylate ${ }^{2}$ (Retardin, Lomotil), with contrary effects on the intestinal function, were administered. Diphenoxylate is a pethidine derivative with a pronounced antidiarrhoeal effect but with a narcotic action that is considerably smaller than that of pethidine and morphine.

At the same time we have examined the extent to which the clinical effects of these drugs are reflected in changes in intraluminal pressure curves and propulsion.

\section{MATERIAL AND METHODS}

Nine healthy volunteers between 20 and 30 years of age were examined on three different occasions: without medication, after the intramuscular injection of Prostigmine, and after the oral administration of diphenoxylate. Tests on a particular subject were performed at intervals of about one week and in random order.

The endoradiosonde used in this study was the P 2 type,

'This investigation was supported by research grant no. A-2338 from The National Institute of Arthritis and Metabolic Diseases, U.S. Public Health Service.

${ }^{2}$ Diphenoxylate tablets were kindly supplied by AB Leo, Hälsingborg, Sweden. described by Jacobson and Nordberg (1961). We employed the modification with a release mechanism by which the recording in the uppermost part of the small intestine could be started at a desired time. The endoradiosonde was not enclosed in a limp bag, as is the usual practice in other laboratories, and was thus insensitive to direct squeezing by the gut; it measured true intraluminal pressure only.

The signal from the endoradiosonde was intercepted by a receiver described by Jacobson and Lindberg (1960). To follow its propulsion through the small intestine an antenna servo system was used which automatically tracks the sonde during its passage through the alimentary canal and charts its movements (Jacobson, 1961). The

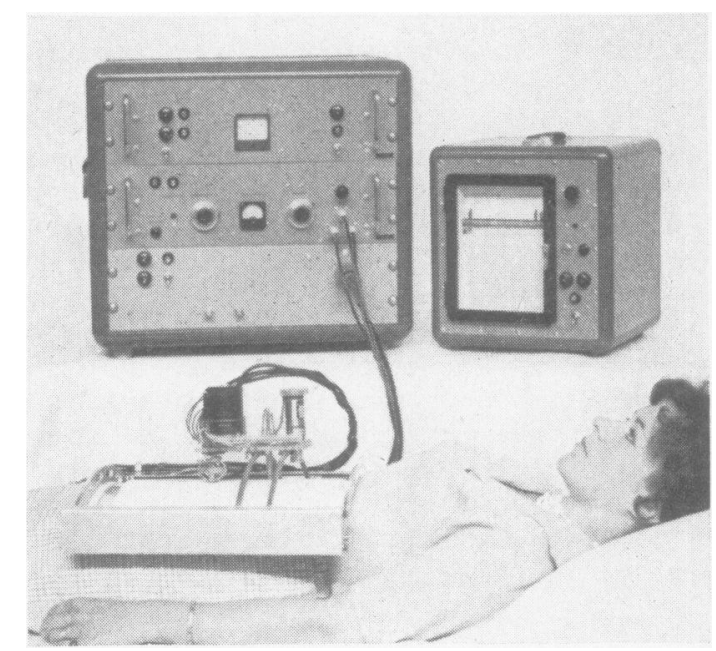

FIG. 1. Automatic tracking and receiving system for interception of the signal transmitted by the endoradiosonde. The servo-controlled antenna carriage charts the movements of the transmitter. 
servoed tracker records movements in the cranio-caudal and right-left directions, but not in the dorso-ventral direction (Fig. 1).

The endoradiosonde, fastened to a plastic tube with the release mechanism, was swallowed on the evening before the experiment. By eight o'clock the next morning the endoradiosonde had usually passed the pylorus; if not, it was withdrawn and the experiment repeated another day. When the experiment could be pursued, a standard meal consisting of bread, butter, and milk was given, and immediately afterwards the endoradiosonde was released from the plastic tube, which was then withdrawn. The intraluminal pressure and the propulsion of the sonde were recorded almost continuously for at least five hours. Another meal was given four hours after the first without interrupting the recording.

In the Prostigmine experiments a dose of 0.5 to $1.0 \mathrm{mg}$. of the drug was injected 90 minutes after the endoradiosonde had been released. By this time the intestinal activity due to the first meal had ceased, as judged from the pressure curves and propulsion. The diphenoxylate was given orally shortly before the first meal in a dose of $5 \mathrm{mg}$. The same dose was given a second time two hours after the first meal.

\section{ANALYSES OF DATA}

The pressure waves recorded by endoradiosondes are identical with those obtained from the same site by means of open-ended tubes (Farrar and Bernstein, 1958; Connell and Rowlands, 1960). Two types of waves are predominant in the small intestine: type I, which are waves of short duration, often occurring in a rhythmic pattern with a frequency of 8 to 12 contractions per minute; and type III waves, which consist of a rise of basic pressure, with superimposed type I peaks (Code, Hightower, and Morlock, 1952). The rise in basic pressure is not rhythmic and of varying duration, although always longer than that of the type I waves.

For the purpose of analysis the curves were divided into 15 -minute periods. For each period the sum of the amplitudes (in centimetres of water) of the type I waves was calculated, including the type I peaks superimposed on the type III waves. A measure of the rise in basic pressure of the type III waves was obtained by planimetry of the total area formed by the lower envelope of the pressure curve and the base-line for each 15-minute period. The rise in basic pressure was calculated separately because its physiological significance would seem to differ from that of the rapid variations.

The following quantities for each 15-minute period were also calculated: the number of pressure peaks and the average amplitude of the type I waves; the total number, the average duration, and the mean base-line rise of the type III waves. These results are not reported here since they did not provide information beyond that obtained from data already reported.

The propulsion was measured with a map meter on the chart drawn by the servoed tracker. Since the tracings for different time intervals were superimposed, frequent change of the paper was necessary. Displacements smaller than 1 centimetre have been disregarded since this is the limit of accuracy of the tracing system, and, in any case, such small displacements can result from breathing and other irrelevant movements of the viscera.

\section{RESULTS}

Test meals and Prostigmine produced an enhanced type I and type III activity. For patients who had not received diphenoxylate the general pressure wave pattern after these two stimuli is very similar; the pattern has an irregular appearance with pressure peaks of greatly varying amplitude (Fig. 2). When diphenoxylate was administered the test meals often produced extended bursts of type I activity with high and fairly constant amplitude (Fig. 3). Such bursts were also seen in the periods between meals, although less frequently. The enhanced type I activity after diphenoxylate was always accompanied by an increased number of type III waves. In a few cases the described effect of diphenoxylate could not be recognized; as a matter of fact a slightly decreased pressure activity was seen in three of the nine diphenoxylate experiments.
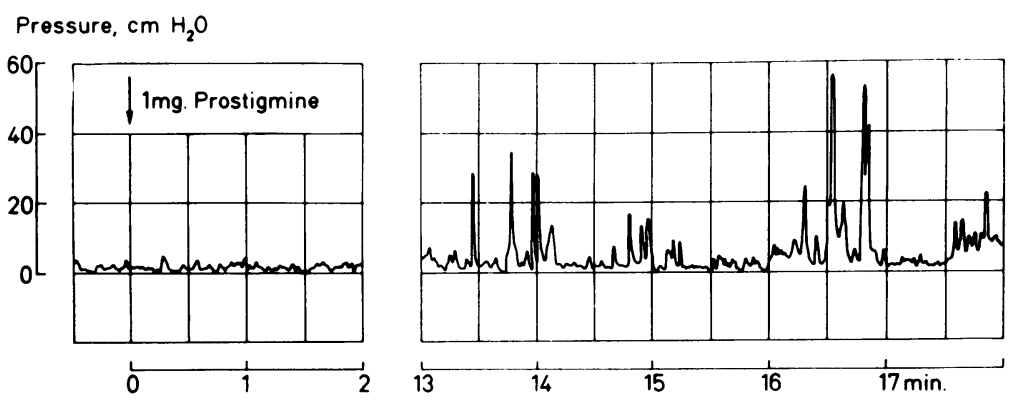

FIG. 2. The effect of $1 \mathrm{mg}$. Prostigmine, administered intramuscularly, on the pressure curve for the small intestine. 
Pressure, $\mathrm{cm} \mathrm{H} \mathrm{H}_{2} \mathrm{O}$

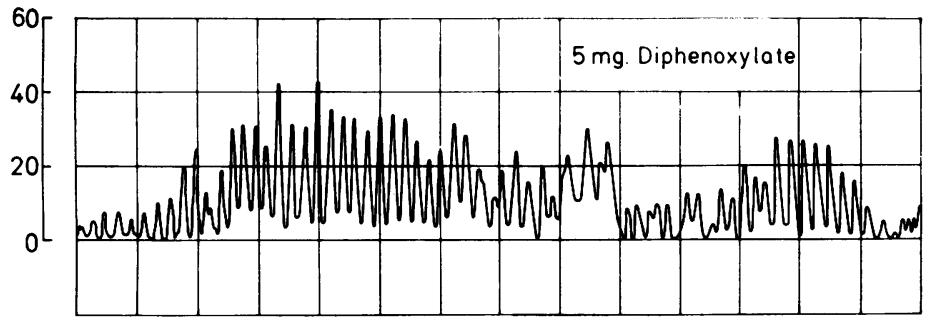

FIG. 3. Pressure curves for the jejunum of a patient on two occasions with and without diphenoxylate, given orally. Both curves were taken during the second 15-minute period after the first test meal. The effect

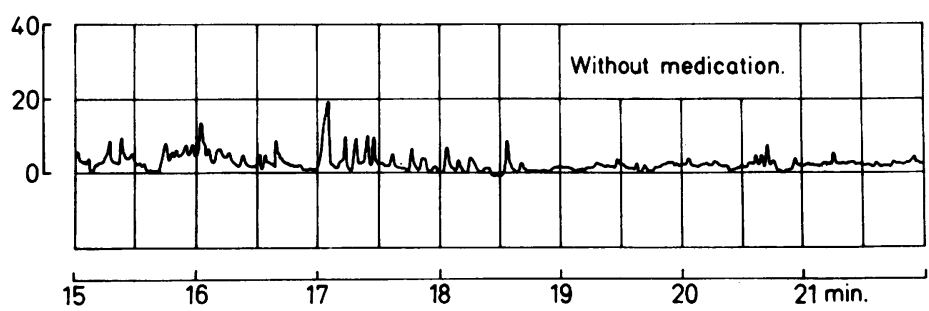
of the drug is usually less pronounced than is seen here and may not be evident in all patients.

The results of a representative experiment are given in Figure 4. Each column represents the pressure activity over 15 minutes. There was a definite increase in activity after the standard meals. The corresponding chart for the propulsion of the endoradiosonde is shown in Figure 5.

A detailed comparison of the individual pressure curves and the corresponding charts of propulsion disclosed no true synchronism between either type of

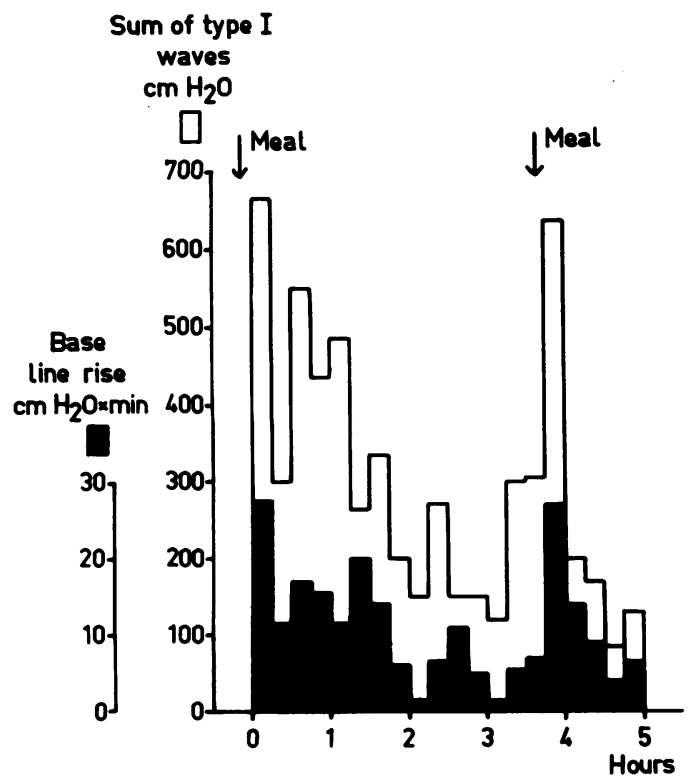

FIG. 4. Diagram of the pressure activity during 15minute periods for a healthy subject during passage of the endoradiosonde from the duodenum to the ileocaecal valve. pressure activity and the movement of the endoradiosonde. On the contrary, a rapid movement of the endoradiosonde was often unaccompanied by any pressure waves at all. This was also the case after the first test meal, when the endoradiosonde passed through the duodenum and upper jejunum and rapid propulsion usually occurred during a period with high pressure activities.

Figure 6 shows the average pressures recorded for

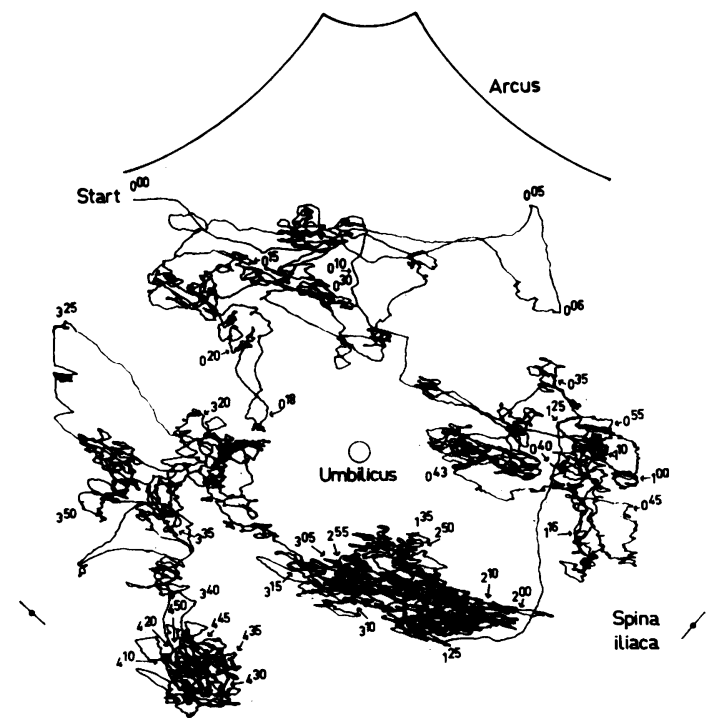

FIG. 5. Chart showing the propulsion of the endoradiosonde from the duodenum to the ileocaecal valve in the same experiment as in Figure 4. The figures refer to the time in hours and minutes after the release of the endoradiosonde in the duodenum. 

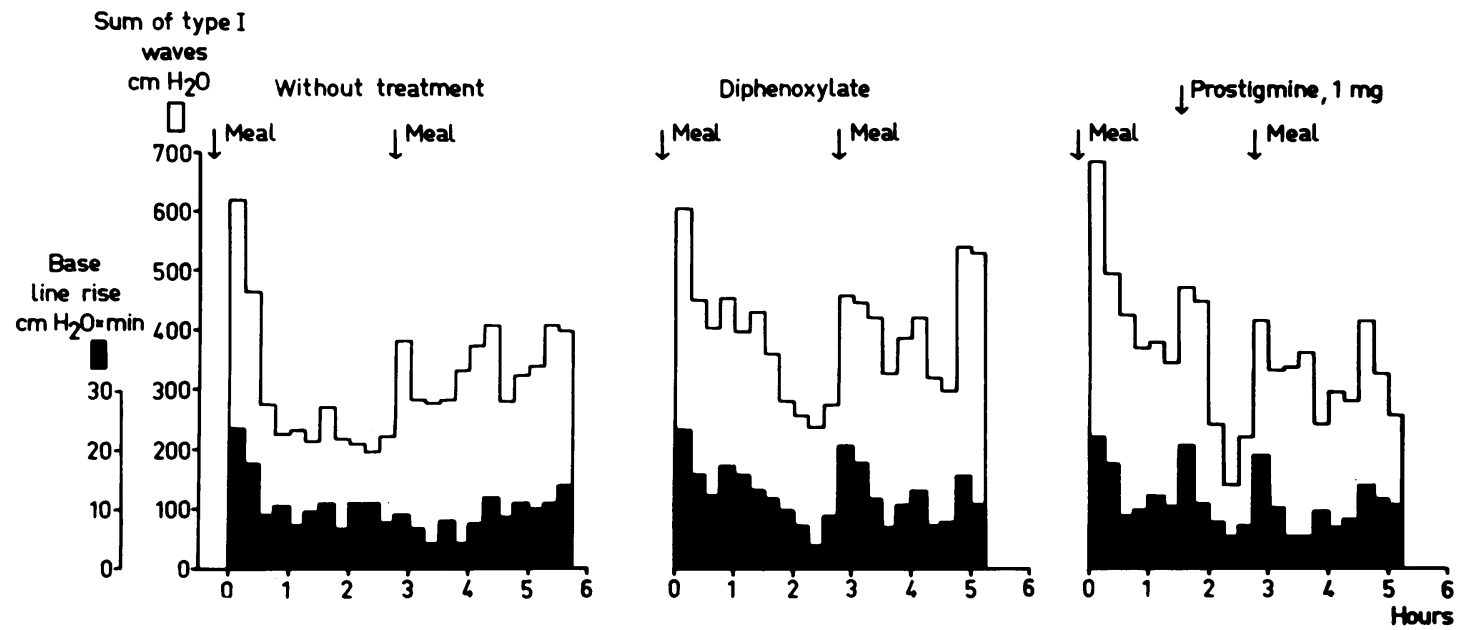

FIG. 6. Diagram of the average pressure activities during 15-minute periods for nine healthy subjects during passage of the endoradiosonde through the small intestine.

the nine subjects in the three experiments. In all instances there was an increase in the pressure activities after the test meals-similar for type I activity and base-line rise. Prostigmine caused an increase in activity, which lasted for about 30 minutes when the dose was $1 \mathrm{mg}$. (With smaller doses little if any effect was observed.) The diagram for diphenoxylate does not differ greatly from that without treatment, the slightly higher level for diphenoxylate being due to a more frequent increase than decrease in activities for the different cases.

The propulsion charts vary so much from one subject to another that averages would be meaningless. Instead, an individual comparison has been made between the pressure activities for 15-minute periods and the propulsion of the endoradiosonde, as measured on the charts. The first test meal was accompanied by high pressure activities and rapid propulsion, an effect that was not altered by diphenoxylate (Fig. 7). No statistically significant correlation between the pressure activities and propulsion was found after the first test meal.

When the second test meal was given the endoradiosonde was located in the ileum or lower part of the jejunum. This meal usually caused increased pressure activities but less than the first test meal. In some cases a prompt propulsive movement of the endoradiosonde was also seen after the second meal but the average propulsion during the first two 15-minute periods after the meal was similar to that before the meal. Diphenoxylate seemed to depress the propulsion after the second test meal.

During the period between meals (at least 90 minutes after the first meal) there was no evident correlation between the pressure activities and propulsion (Fig. 7). After diphenoxylate the propulsion was slower than where there was no medication, whereas the pressure activities were of the same magnitude, or even enhanced.

The increase in the pressure activities during the first two 15-minute periods after the administration of Prostigmine corresponded to an acceleration of the propulsion (Fig. 7).

\section{DISCUSSION}

The effects of the test meals and of Prostigmine recorded by the endoradiosonde bear out those observed with other techniques (Kewenter and Kock, 1960). Both these stimuli resulted in an increase in pressure activities and propulsion. Diphenoxylate, on the other hand, produced a decrease in propulsion without a corresponding decrease in pressure activities. Thus, it is likely that the constipating effect of diphenoxylate is caused by an increased tonus and diminished calibre of the intestine as has been suggested for morphine (Benczur, 1910; Plant and Miller, 1926).

The fact that diphenoxylate can give in some cases an increase and in others a decrease in pressure activities can be explained by the assumption that the drug has two antagonistic effects on the small intestine. This is not unlikely since morphine is known to have both a stimulating and a paralysing effect, depending on the dose and the site of action (Nothnagel, 1884; Krueger, Howes, and Gay, 1935).

It is generally accepted that type I waves correspond to the rhythmic segmentation of the small intestine. This type of motility has very little propulsive action (Cannon, 1911). The increase in type I activity and propulsion recorded after the first test 
FIG. 7. Propulsion of the endoradiosonde during 15-minute periods, plotted as a function of the corresponding pressure activities. 
meal is not incompatible with this view, since no statistical correlation between the two entities was found. Nor was there any evidence of a synchronism between the individual pressure peaks and the propulsion of the endoradiosonde. The results obtained with and without diphenoxylate after the second meal and between meals, when propulsion seemed to be completely unrelated to the type I activity, confirm the view that the type I waves have no propulsive action.

The possibility of a connexion between the type III waves and propulsion has received much attention. Some investigators using balloon and $x$-ray techniques have found a synchronism between the propulsion of the intestinal content and the occurrence of a type III wave (Chapman and Palazzo, 1949; Glazebrook, 1956). With the endoradiosonde technique we have not been able to confirm this view. No true synchronism and no quantitative correlation was found between type III waves and propulsion in the experiments without treatment and with diphenoxylate. In the Prostigmine experiments, however, a correlation between propulsion and both types of pressure activity might exist, but the number of 15-minute periods studied was too small for a statistical analysis to have validity. Here, too, there was no synchronism between individual pressure waves and propulsion of the endoradiosonde. The drug may well cause an increase in propulsion and pressure activities independently of one another. It may be recalled here that we have been able to record propulsion in two directions only, which increases the scatter of the values.

The reason why the propulsion of an endoradiosonde is not related to the type III waves in our experiments might either be that these waves do not have the assumed propulsive action, or that the endoradiosonde is processed differently from the rest of the intestinal content. The latter possibility might be correct, considering the observations by Benczur (1910), who found that the propulsion of a large bolus becomes considerably slower than that of a small bolus when the calibre of the gut decreases. Thus, a correlation between the type III waves and propulsion of the ordinary intraluminal content may well exist in spite of our negative results.

It might be wondered, on the other hand, why large extended pressure waves, such as the type III waves, should be necessary to move a fluid intestinal content, when small pressure gradients of short duration would suffice. The relationship between propulsion and pressure activity calls for study by more refined methods.

\section{SUMMARY}

Nine healthy volunteers were examined with an endoradiosonde method for recording the intraluminal pressure of the small intestine. At the same time the propulsive movement of the sonde was charted by an automatic tracking device. Measurements were made continuously for five hours. Test meals and Prostigmine were found to cause an increase in the number and the amplitude of the type I waves and a rise in basic pressure of the type III waves. Giving diphenoxylate, which has a constipating effect, usually resulted in an increase, but sometimes a decrease, in both types of pressure activities. During the enhanced piessure activities after a test meal with an endoradiosonde in the upper part of the intestine, and after Prostigmine, there was an acceleration of the endoradiosonde, but there was no evidence of synchronism between any type of pressure peaks and the propulsive movement. In the distal part of the small intestine there was no apparent relationship between the pressure activities and propulsion of the endoradiosonde. Some of the findings are difficult to understand on the basis of the generally accepted view that the type III waves have a propulsive action. They might be explained, though, by a different propagation of a solid body of the size of the endoradiosonde as compared with a fluid or soft intestinal content.

\section{REFERENCES}

Benczur, G. von (1909-10). Beiträge zur Kenntnis der Peristaltik des Dünndarms. Int. Beitr. Ernähr Ston., 1, 5-28.

Cannon, W. B. (1911). The Mechanical Factors of Digestion, pp. 130137. Arnold, London.

Code, C. F., Hightower, N. C., Jr., and Morlock, C. G. (1952), Motility of the alimentary canal in man. Review of recent studies. Amer. J. Med., 13, 328-351.

Connell, A. M. (1961). The motility of the small intestine. Postgrad. med. J., 37, 703-716.

_- and Rowlands, E. N. (1960). Wireless telemetering from the digestive tract. Gut. 1, 266-272.

Chapman, W. P., and Palazzo, W. L. (1949). Multiple-balloonkymograph recording of intestinal motility in man with observations on the correlation of the tracing patterns with barium movements. J. clin. Invest., 28, 1517-1525.

Farrar, J. T., and Bernstein, J. S. (1958). Recording of intraluminal gastrointestinal pressures by a radiotelemetering capsule. Gastroenterology, 35, 603-612.

Glazebrook, A. J. (1956). Jejunal motility patterns. Canad. med. Ass. J., 75, 642-650.

Jacobson, B. (1961). Servoed antenna tracks radio pill. Contr. Engin., 8, no. $12,119$.

- and Lindberg, B. (1960). FM receiving system for endoradiosonde techniques. IRE Trans. med. Electron., ME-7, 334-339.

, and Nordberg, L. (1961). Endoradiosondes for pressure telemetering. IRE Trans. bio-med. Electron., BME-8, 192-196.

Kewenter, J., and Kock, N. G. (1960). Motility of the human small intestine. Acta chir. scand., 119, 430-438.

Krueger, H., Howes, H. A., and Gay, H. (1935). The effects of morphine and its derivatives on intestinal movements. $J$. Pharmacol. exp. Ther., 55, 288-318.

Nothnagel, C. W. H. (1884). Beiträge zur Physiologie und Pathologie des Darmes, pp. 62-69. Hirschwald, Berlin.

Plant, O. H., and Miller, G. H. (1926). Effects of morphine and some other opium alkaloids on the muscular activity of the alimentary canal. 1. Action on the small intestine in unanaesthetized dogs and man. J. Pharmacol. exp. Ther., 27, 361-383. 